\title{
STRONG AMALGAMATIONS OF LATTICE ORDERED GROUPS AND MODULES
}

\author{
MONA CHERRI \\ Department of Mathematics and Computer Science \\ Texas Woman's University \\ Denton, Texas 76204 \\ and
}

WAYNE B. POWELL

Department of Mathematics

Oklahoma State University

Stillwater, Oklahoma 74078

(Received September 25, 1990 and in revised form January 21, 1992)

\begin{abstract}
We show that every variety of representable lattice ordered groups fails the strong amalgamation property. The same result holds for the variety of $f$-modules over an $f$-ring. However, strong amalgamations do occur for abelian lattice ordered groups or $f$-modules when the embeddings are convex.
\end{abstract}

KEY WORDS AND PHRASES. Amalgamation property, ordered group, ordered module, free product.

1991 AMS SUBJECT CLASSIFICATION CODE. 06F15, 06F25; 08B25.

\section{INTRODUCTION}

In this paper we consider two variations of the amalgamation property for classes of lattice ordered groups (l-groups) and lattice ordered modules. The first of these is the strong amalgamation property which we will show fails in every variety of representable $l$-groups as well as in a particular class of lattice ordered modules. Secondly, we investigate the possibility of amalgamating two $l$-groups with a common convex $l$-subgroup. We show that this is possible in the variety of abelian $l$-groups even if the amalgamation is required to be strong. A similar result holds for the variety of lattice ordered modules generated by the totally ordered modules.

Let $U$ be a class of $l$-groups or lattice ordered modules and let $\Gamma=\left(A, B_{1}, B_{2}, \alpha_{1}, \alpha_{2}\right)$ be a quintuple with $A, B_{1}, B_{2} \in U$ and $\alpha_{1}: A \rightarrow B_{1}$ and $\alpha_{1}: A \rightarrow B_{1}$ and $\alpha_{2}: A \rightarrow B_{2} l$-monomorphisms. Then $\Gamma$ is called a $V$-formation in $U$. The $V$-formation $\Gamma$ can be amalgamated in $U$ if there exists a triple $\left(C, \beta_{1}, \beta_{2}\right)$ such that $C \in U, \beta_{1}: B_{1} \rightarrow C$ and $\beta_{2}: B_{2} \rightarrow C$ are $l$-monomorphisms, and $\beta_{1} \alpha_{1}=\beta_{2} \alpha_{2}$. This is depicted by the following diagram.

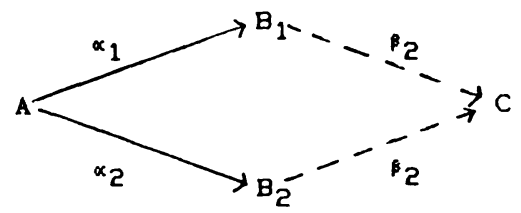


If every $V$-formation in $U$ can be amalgamated in $U$, then $U$ is said to have the amalgamation property (AP). The triple $\left(C, \beta_{1}, \beta_{2}\right)$ is called a strong amalgamation of $\Gamma$ if it amalgamates $\Gamma$ in such a way that $\beta_{1}\left(b_{1}\right)=\beta_{2}\left(b_{2}\right)$ implies $b_{1} \in \alpha_{1}(A)$ and $b_{2} \in \alpha_{2}(A)$. If all $V$-formations in $U$ can be strongly amalgamated, then $U$ is said to have the strong amalgamation property (StAP).

In our subsequent discussion there are several varieties of $l$-groups which will play a significant role. These are given distinguished notation as listed below.

$L=$ variety of all $l$-groups;

$R=$ variety of representable $l$-groups (defined by $\left.(x \wedge y)^{2}=x^{2} \wedge y^{2}\right) ;$ and

$A=$ variety of abelian $l$-groups (defined by $x y=y x$ ).

The variety $A$ is the smallest nontrivial variety of $l$-groups (Weinberg [18]). The representable $l$ groups are important since they are precisely those $l$-groups which are subdirect products of totally ordered groups.

Among the lattice ordered modules there is one class which stands out in its significance. This is the class of $f$-modules, which is the variety generated by all totally ordered modules. This class of modules forms the natural generalization of the important class of vector lattices. In this paper we shall restrict ourselves to lattice ordered modules over rings which are $f$-rings; i.e., that are subdirect products of totally ordered rings. Given such a ring $S$ we let

$$
M=\text { variety of } f \text {-modules over } S \text {. }
$$

The investigation of the amalgamation property for classes of $l$-groups was begun by Pierce in [7], [8], and [9]. Here he showed among other things that the variety $L$ fails AP while the variety of abelian l-groups satisfies this property. Implicit in his work is a proof that the varieties above and including the non-representable covers of $A$ also fail AP. Subsequently, Powell and Tsinakis showed in [12] and [13] that there exists an uncountable chain of varieties containing $R$ and failing AP such that their join is the largest proper variety of $l$-groups. It was later proved by Glass, Saracino, and Wood [4] that the variety $R$ itself and many varieties contained therein cannot have the amalgamation property. In [15] Powell and Tsinakis extended this result to all representable varieties containing one of the two solvable, non-nilpotent covers of $A$. Further, in [12] they showed that the varieties of nilpotent $l$-groups do not satisfy AP. To date no general proof has surfaced to show that AP fails in all nonabelian varieties of $l$-groups although this result is likely to be true.

For basic information on l-group free products and amalgamations, see Powell and Tsinakis [12], [16], and [17]. Background on lattice ordered groups and modules in general can be found in Bigard, Keimel and Wolfenstein [2]. The only paper to date investigating free products of $f$ modules is Cherri and Powell [3], although several papers on free $f$-modules help introduce the subject (see Bigard [1], Powell [10], or Powell and Tsinakis [14]).

\section{THE STRONG AMALGAMATION PROPERTY.}

We will show in this section that any class of representable l-groups containing $Z$ (the integers) and closed with respect to the formation of $l$-subgroups and direct products fails StAP. A similar result follows for a class of $f$-modules containing the ring $S$ and closed with respect to the formation of $l$-submodules and direct products. Our initial effort will be with $l$-groups, and we will subsequently point out the analogous proofs for modules. The first step is to relate amalgamations to free products in the given class. General existence theorems guarantee that these structures can be considered in the classes we are examining (see Grätzer [5]).

To avoid repetition of hypotheses we will make some standing definitions here. 
i) $U$ is a class of $l$-groups containing $\mathbf{Z}$ and closed with respect to the formation of $l$-subgroups and direct products;

ii) $\Gamma=\left(A, B_{1}, \alpha_{1}, \alpha_{2}\right)$ is a $V$-formation in $U$;

iii) $B_{1}\lfloor\rfloor B_{2}$ is the free product of $B_{1}$ and $B_{2}$ in $U$;

iv) $\lambda_{1}: B_{1} \rightarrow B_{1}\lfloor\rfloor B_{2}$ and $\left.\left.\lambda_{2}: B_{2} \rightarrow B_{1}\right\rfloor\right\rfloor B_{2}$ are the natural embeddings;

v) $\quad N$ is the $l$-ideal of $B_{1}\lfloor\rfloor B_{2}$ generated by $\left\{\lambda_{1} \alpha_{1}(a) \lambda_{2} \alpha_{2}(a)^{-1} \mid a \in A\right\}$; and

vi) $\sigma: B_{1}\lfloor\rfloor B_{2} \rightarrow B_{1}\lfloor\rfloor B_{2} / N$ is the natural projection.

The first Lemma puts amalgamations in terms of free products.

LEMMA 1 . If $\Gamma$ can be strongly amalgamated in $U$, then it can be strongly amalgamated by the triple $\left(B_{1}\lfloor\rfloor B_{2} / N, \sigma \lambda_{1}, \sigma \lambda_{2}\right)$.

PROOF. Suppose that $\Gamma$ can be amalgamated by the triple $\left(C, \beta_{1}, \beta_{2}\right)$ in $U$. Then there is a natural map $\eta: B_{1}\lfloor\rfloor B_{2} \rightarrow C$ extending the maps $\beta_{1}$ and $\beta_{2}$. From the relationship $\beta_{1} \alpha_{1}=\beta_{2} \alpha_{2}$ it is clear that $N \subseteq$ ker $\eta$. Hence, there exists a map $\rho: B_{1}\lfloor\rfloor B_{2} / N \rightarrow C$ such that $\rho \sigma=\eta$. From this it follows that $\left.\left.\left(B_{1}\right\rfloor\right\rfloor B_{2} / N, \sigma \lambda_{1}, \sigma \lambda_{2}\right)$ amalgamates $\Gamma$ in $U$. This is depicted in the following commutative diagram.

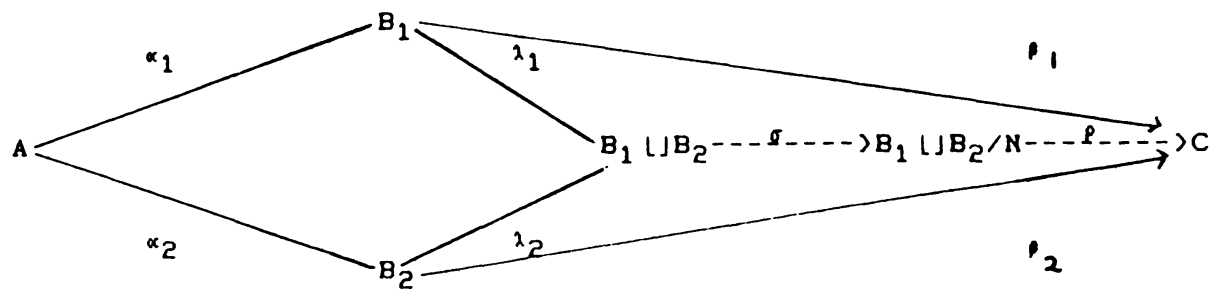

Now to see that this new amalgamation is indeed strong suppose that $\sigma \lambda_{1}\left(b_{1}\right)=\sigma \lambda_{2}\left(b_{2}\right)$ for some $b_{1} \in B_{1}$ and $b_{2} \in B_{2}$. Then $\beta_{1}\left(b_{1}\right)=\rho \sigma \lambda_{1}\left(b_{1}\right)=\rho \sigma \lambda_{2}\left(b_{2}\right)=\beta_{2}\left(b_{2}\right)$. By the properties of $C$ this implies that there exists $a \in A$ such that $\alpha_{1}(a)=b_{1}$ and $\alpha_{2}(a)=b_{2}$.

The preceding Lemma is all that is necessary to examine strong amalgamations in $U$.

THEOREM 2. If $U \subseteq R$, then $U$ fails StAP.

PROOF. In general let $(x)$ denote the totally ordered cyclic group generated by $x$. Set $A=(a), B_{1}=\left(b_{1}\right)$, and $B_{2}=\left(b_{2}\right)$. Define

$$
\alpha_{1}: A \rightarrow B_{1} \text { and } \alpha_{2}: A \rightarrow B_{2}
$$

by

$$
\alpha_{1}(a)=b_{1}^{2} \text { and } \alpha_{2}(a)=b_{2}^{2} .
$$

If $\Gamma=\left(A, B_{1}, B_{2}, \alpha_{1}, \alpha_{2}\right)$ can be strongly amalgamated in $U$, then this can be done by $B_{1}\lfloor\rfloor B_{2} / N$ where our notation is the same as has been previously used. By the nature of $N$ we have $\lambda_{1} \alpha_{1}(a) \lambda_{2} \alpha_{2}(a)^{-1} \in N$ so $\lambda_{1}\left(b_{1}^{2}\right) \lambda_{2}\left(b_{2}^{2}\right)^{-1} \in N$. Now $B_{1}\lfloor\rfloor B_{2}$ is in $U \subseteq R$ so it is a subdirect product of totally ordered groups each of which is also in $U$. On each of these totally ordered groups, either

$$
\lambda_{1}\left(b_{1}\right) \geq \lambda_{2}\left(b_{2}\right) \geq 1 \text { or } \lambda_{2}\left(b_{2}\right) \geq \lambda_{1}\left(b_{1}\right) \geq 1 .
$$

In the first case we have $\lambda_{1}\left(b_{1}\right) \lambda_{2}\left(b_{2}\right)^{-1} \geq 1$ and $\lambda_{2}\left(b_{2}\right)^{-1} \lambda_{1}\left(b_{1}\right) \geq 1$. This implies that

$$
\begin{aligned}
\lambda_{1}\left(b_{1}\right) \lambda_{2}\left(b_{2}\right)^{-2} \lambda_{1}\left(b_{1}\right) & =\left[\lambda_{1}\left(b_{1}\right) \lambda_{2}\left(b_{2}\right)^{-1}\right]\left[\lambda_{2}\left(b_{2}\right)^{-1} \lambda_{1}\left(b_{1}\right)\right] \\
& \geq \lambda_{2}\left(b_{2}\right)^{-1} \lambda_{1}\left(b_{1}\right) \\
& \geq \lambda_{1}\left(b_{1}\right)^{-1} \lambda_{2}\left(b_{2}\right) .
\end{aligned}
$$


On the other hand, if $\lambda_{2}\left(b_{2}\right) \geq \lambda_{1}\left(b_{1}\right) \geq 1$, then $\lambda_{2}\left(b_{2}\right) \lambda_{1}\left(b_{1}\right)^{-1} \geq 1$ and $\lambda_{1}\left(b_{1}\right)^{-1} \lambda_{2}\left(b_{2}\right) \geq 1$. In this case we have

$$
\begin{aligned}
\lambda_{1}\left(b_{1}\right)^{-1} \lambda_{2}\left(b_{2}\right)^{2} \lambda_{1}\left(b_{1}\right)^{-1} & =\left[\lambda_{1}\left(b_{1}\right)^{-1} \lambda_{2}\left(b_{2}\right)\right]\left[\lambda_{2}\left(b_{2}\right) \lambda_{1}\left(b_{1}\right)^{-1}\right] \\
& \geq \lambda_{1}\left(b_{1}\right)^{-1} \lambda_{2}\left(b_{2}\right) \\
& \geq \lambda_{2}\left(b_{2}\right)^{-1} \lambda_{1}\left(b_{1}\right) .
\end{aligned}
$$

Together these last two inequalities mean that in $B_{1}\lfloor\rfloor B_{2}$ we have

$$
\begin{aligned}
\left|\lambda_{1}\left(b_{1}\right) \lambda_{2}\left(b_{2}\right)^{-2} \lambda_{1}\left(b_{1}\right)\right| & =\left[\lambda_{1}\left(b_{1}\right) \lambda_{2}\left(b_{2}\right)^{-2} \lambda_{1}\left(b_{1}\right)\right] \vee\left[\lambda_{1}\left(b_{1}\right)^{-1} \lambda_{2}\left(b_{2}\right)^{2} \lambda_{1}\left(b_{1}\right)^{-1}\right] \\
\geq & {\left[\lambda_{1}\left(b_{1}\right)^{-1} \lambda_{2}\left(b_{2}\right)\right] \vee\left[\lambda_{2}\left(b_{2}\right)^{-1} \lambda_{1}\left(b_{1}\right)\right] } \\
\geq & \lambda_{2}\left(b_{2}\right)^{-1} \lambda_{1}\left(b_{1}\right) .
\end{aligned}
$$

But $\lambda_{1}\left(b_{1}\right)^{2} \lambda_{2}\left(b_{2}\right)^{-2} \in N$ and so by normality $\lambda_{1}\left(b_{1}\right) \lambda_{2}\left(b_{2}\right)^{-2} \lambda_{1}\left(b_{1}\right) \in N$. The preceding inequality together with the convexity of $N$ implies that $\lambda_{2}\left(b_{2}\right)^{-1} \lambda_{1}\left(b_{1}\right) \in N$. Finally, we have $\sigma \lambda_{1}\left(b_{1}\right)=\sigma \lambda_{2}\left(b_{2}\right)$ while $b_{1} \notin \alpha_{1}(A)$ and $b_{2} \notin \alpha_{2}(A)$, contradicting the strongness of the amalgamation.

The preceding proof also applies to the variety $M$ of $f$-modules over an $f$-ring $S$. The only difference in the proof for the module case is that $S$ is substituted for $\mathbf{Z}$.

THEOREM 3. The variety $M$ of $f$-modules over an $f$-ring $S$ fails the strong amalgamation property.

Whereas varieties of $l$-groups have been investigated in some detail with regard to the (general) amalgamation property, the $f$-modules have not yet received such attention. In Cherri and Powell [3] the free products within such classes are considered in detail. Among other things it is shown there that the special amalgamation property is satisfied by using a representation of the free products. This, together with the congruence extension property, implies that the class $M$ does in fact satisfy the amalgamation property (Grätzer and Lakser [6]).

3. CONVEX AMALGAMATIONS.

In the proof of Theorem 2, the image of $A$ was not convex in $B_{1}$ or $B_{2}$. If we consider $V$ formations where this is the case, we find that strong amalgamations can occur. In fact for the variety $A$ of abelian $l$-groups it will always be possible. A similar situation holds for the variety $M$ of $f$-modules if the ring $S$ is assumed to be totally ordered and a left Ore domain. These assumptions on $S$ are used in creating the representation of $M$-free products described below.

The proof of the next theorem will draw on representations of free products in $A$ and $M$. We describe briefly this process here and refer the reader to Powell and Tsinakis [11] and Cherri and Powell [3] for details. Let $G$ and $H$ be abelian $l$-groups (or $f$-modules in $M$ ) and consider the sets $\left\{P_{i} \mid i \in I\right\}$ and $\left\{Q_{j} \mid j \in J\right\}$ of primes of $G$ and $H$, respectively. For each $i \in I$ and $j \in J$ there is at least one total order $T$ on $G / P_{i} \times H / Q_{j}$ which extends the orders on $G / P_{i}$ and $H / Q_{j}$. Let $\Lambda=\Pi\left(G / P_{i} \times H / Q_{j}, T\right)$ be the direct product of all such totally ordered groups (respectively, modules), where the product is taken over all $i \in I, j \in J$, and appropriate total orders $T$. Then there is a natural embedding $\gamma: G \times H \rightarrow \Lambda$ of the group $G \times H$, and $G\lfloor\rfloor H$ is the sublattice of $\Lambda$ generated by $\gamma(G \times H)$. That is,

$$
G\lfloor\rfloor H=\left\{\bigvee_{k} \wedge \hat{n} \gamma\left(x_{k n}\right) \mid x_{k n} \in G \times H\right\} .
$$

The terms in the product, that is the terms of the form $\left(G / P_{i} \times H / Q_{j}, T\right)$, are called the components of $G\lfloor\rfloor H$. 
In the following theorem the notation previously established is continued.

THEOREM 4. If $\Gamma$ is a $V$-formation in $A$ or $M$ and if $\alpha_{1}(A)$ and $\alpha_{2}(A)$ are convex in $B_{1}$ and $B_{2}$, respectively, then $\Gamma$ can be strongly amalgamated.

PROOF. We consider a $V$-formation in $A$, leaving the completely analogous proof for $M$ to the reader. We know that an amalgamation of $\Gamma$ exists in $A$ since this variety has AP. To see that this amalgamation can be made strong we consider $B_{1}\lfloor\rfloor B_{2} / N$ and the maps $\sigma \lambda_{1}: B_{1} \rightarrow B_{1}\lfloor\rfloor B_{2} / N$ and $\sigma \lambda_{2}: B_{2} \rightarrow B_{1}\lfloor\rfloor B_{2} / N$. Suppose now that $b_{1} \in B_{1}$ and $b_{2} \in B_{2}$ where $b_{1} \notin \alpha_{1}(A)$. We must show that $\sigma \lambda_{1}\left(b_{1}\right) \neq \sigma \lambda_{2}\left(b_{2}\right)$. Since we are dealing with abelian groups, the l-ideal $N$ of $B_{1}\lfloor\rfloor B_{2}$ is just the convex sublattice generated by elements of the form $\lambda_{1} \alpha_{1}(a) \lambda_{2} \alpha_{2}(a)^{-1}$ where $a \in A$. Let $P$ be a prime subgroup of $B_{1}$ such that $\alpha_{1}(A) \subseteq P$ and $b_{1} \notin P$, and let $Q$ be a prime subgroup of $B_{2}$ with $\alpha_{2}(A) \subseteq Q$. Consider the $\left(B_{1} / P \times B_{2} / Q, T\right)$ component in the representation of $B_{1}\lfloor\rfloor B_{2}$ where $T$ is any appropriate total order. Then $\lambda_{1} \alpha_{1}(a) \lambda_{2} \alpha_{2}(a)^{-1}$ is the identity element on this component for any $a \in A$. However, $\lambda_{1}\left(b_{1}\right) \lambda_{2}\left(b_{2}\right)^{-1}$ is nontrivial on this component since $b_{1} \notin P$. But this means that $\lambda_{1}\left(b_{1}\right) \lambda_{2}\left(b_{2}\right)^{-1} \notin N$ and so $\sigma \lambda_{1}\left(b_{1}\right) \neq \sigma \lambda_{2}\left(b_{2}\right)$.

ACKNOWLEDGEMENT. The first author's research was partially supported by a grant from Texas Woman's University. The second author's research was partially supported by a National Science Foundation (EPSCOR) grant.

\section{REFERENCES}

1. BIGARD, A., "Free lattice-ordered modules", Pacific J. Math. 49(1973), 1-6.

2. BIGARD, A., KEIMEL, K. and WOLFENSTEIN, S., Groupes et Anneaux Reticules, SpringerVerlag, Berlin, 1977.

3. CHERRI, M. and POWELL, W.B., "Free products of lattice ordered modules", preprint.

4. GLASS, A.M.W., SARACINO, D., and WOOD, C., "Non-amalgamation of ordered groups", Math. Proc. Camb. Phil. Soc. 95 (1984), 191-195.

5. GRÄTZER, G., General Lattice Theory, Academic Press, New York, 1978.

6. GRÄTZER, G. and LAKSER, H., "The structure of pseudocomplemented distributive lattices. II. Congruence extension and amalgamation", Trans. Amer. Math. Soc. 172 (1971), 343-358.

7. PIERCE, K.R., "Amalgamations of lattice ordered groups", Trans. Amer. Math. Soc. $\underline{172}(1972), 249-260$.

8. PIERCE, K.R., "Amalgamating abelian ordered groups", Pacific J'. Math. 43 (1972), 711-723.

9. PIERCE, K.R., “Amalgamated sums of abelian l-groups", Pacific J. Math. 65 (1976), 167-173.

10. POWELL, W.B., "Projectives in a class of lattice ordered modules", Algebra Universalis $\underline{13}$ (1981), 24-40.

11. POWELL, W.B. and TSINAKIS, C., "Free products in the class of abelian l-groups", Pacific J. Math. 104 (1983), 429-442.

12. POWELL, W.B. and TSINAKIS, C., "Free products of lattice ordered groups", Algebra Universalis 18 (1984), 178-198.

13. POWELL, W.B. and TSINAKIS, C., "Amalgamations of lattice ordered groups", Ordered Algebraic Structures, Marcel Dekker, 1985, 171-178.

14. POWELL, W.B. and TSINAKIS, C., "Disjoint sets in free lattice ordered modules", Houston J. Math., 15 (1989), 417-424.

15. POWELL, W.B. and TSINAKIS, C., "The failure of the amalgamation property in varieties of representable l-groups", Math. Proc. Camb. Phil. Soc., 106 (1989) 439-443. 
16. POWELL, W.B. and TSINAKIS, C., "Amalgamations of lattice ordered groups", Lattice Ordered Groups, Klüwer Acad. Publ., 1989, 308-327.

17. POWELL, W.B. and TSINAKIS, C., "Free products in varieties of $l$-groups", Lattice Ordered Groups, Klüwer Acad. Publ. 1989, 278-307.

18. WEINBERG, E.C., "Free lattice-ordered abelian groups", Math. Ann. 151(1963), 187-199. 


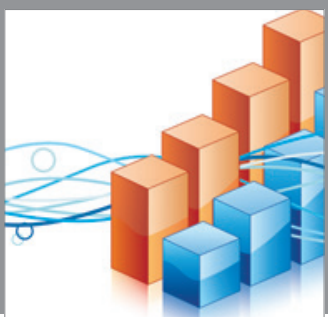

Advances in

Operations Research

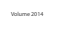

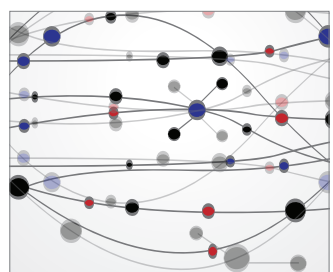

\section{The Scientific} World Journal
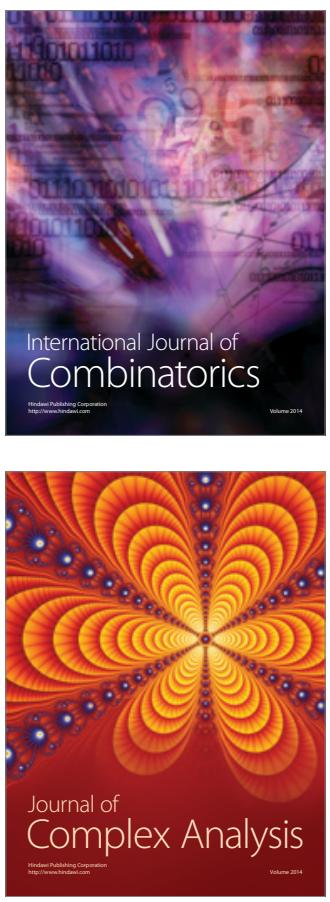

International Journal of

Mathematics and

Mathematical

Sciences
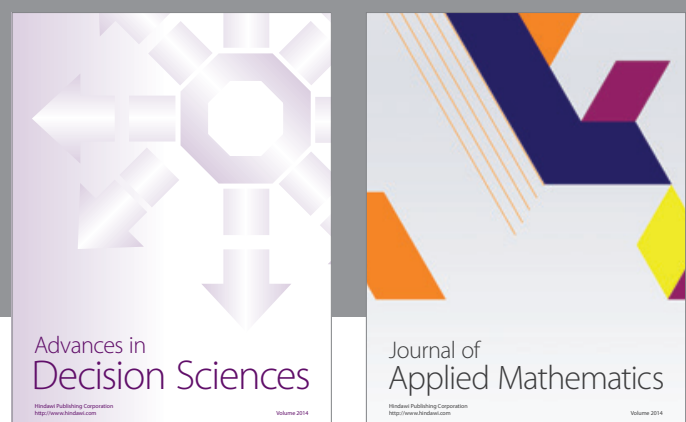

Journal of

Applied Mathematics
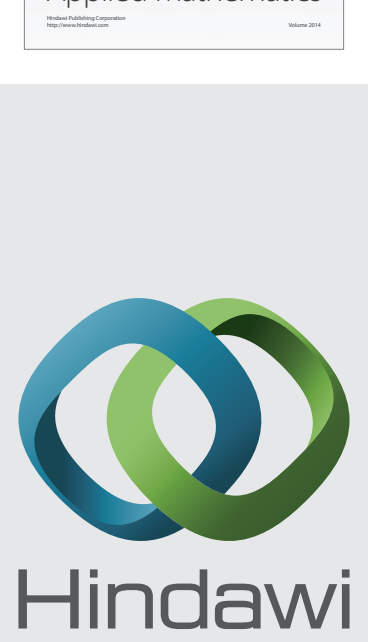

Submit your manuscripts at http://www.hindawi.com
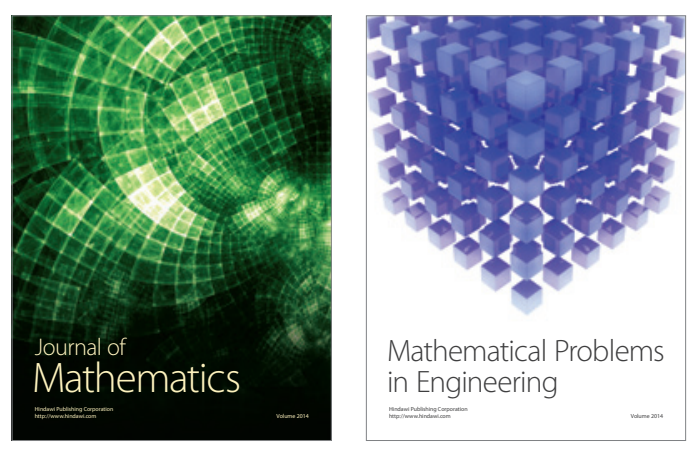

Mathematical Problems in Engineering
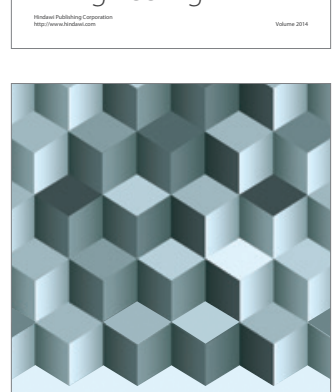

Journal of

Function Spaces
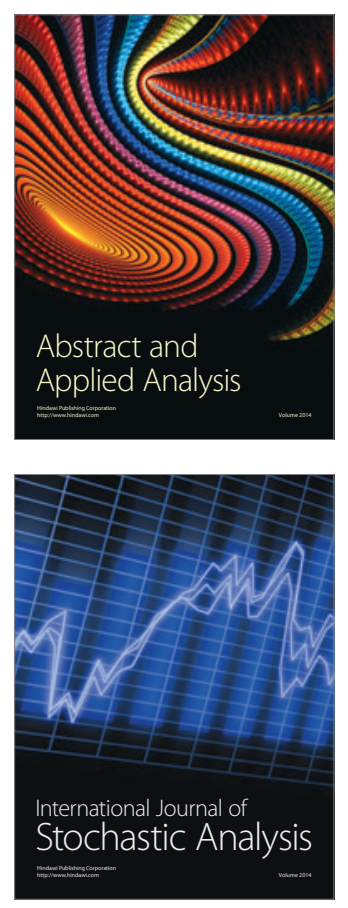

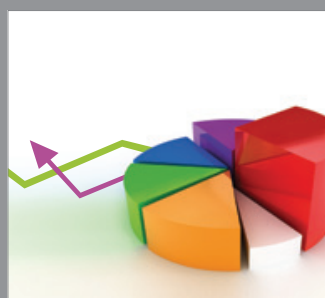

ournal of

Probability and Statistics

Promensencen
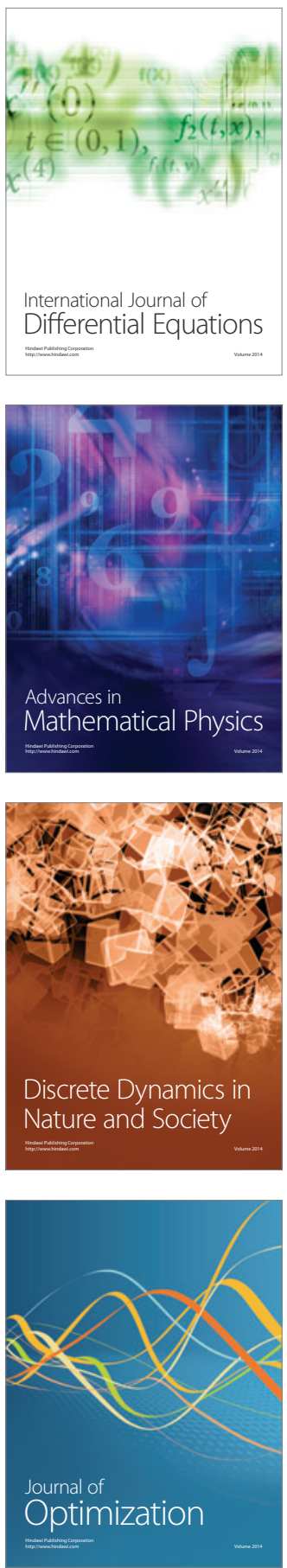